\title{
Use of ultra-short columns for therapeutic protein separations, Part 2: Designing the optimal column dimension for reversed phase liquid chromatography
}

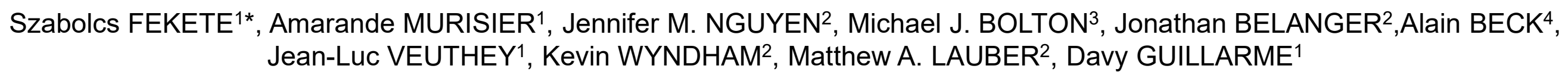

\footnotetext{
1 Institute of Pharmaceutical Sciences of Western Switzerland, University of Geneva, CMU-Rue Michel Servet 1, 1211 Geneva 4, Switzerland

2Waters Corporation, 34 Maple Street, Milford, MA 01757-3696, United States

${ }^{3}$ Sensata Technologies, 529 Pleasant St, Attleboro, MA 027030-2421, United States

${ }^{4}$ Center of Immunology Pierre Fabre, 5 Avenue Napoléon III, BP 60497, 74160 Saint-Julien-en-Genevois, France
}

\section{Supplementary figures, tables}


(A)
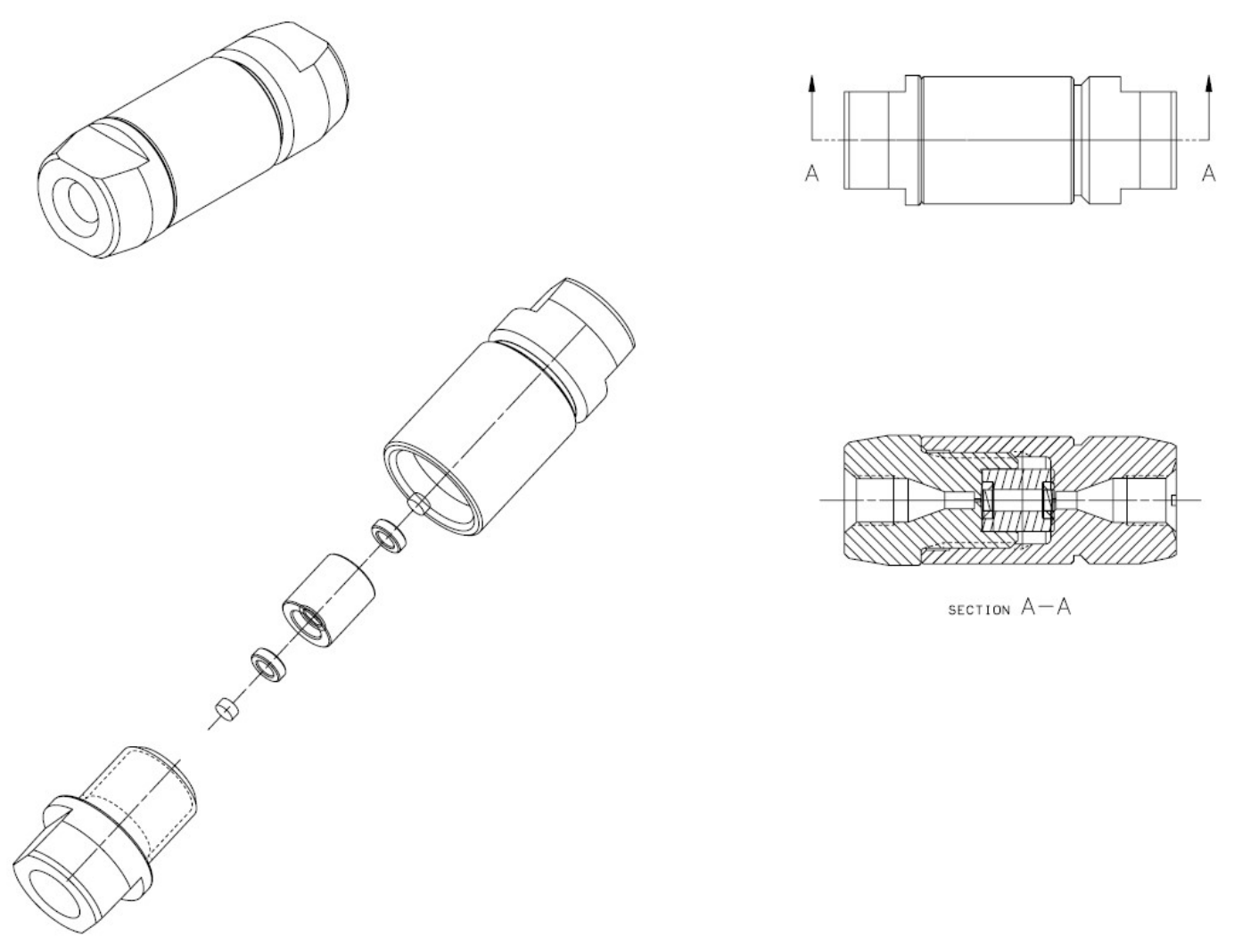

(B)

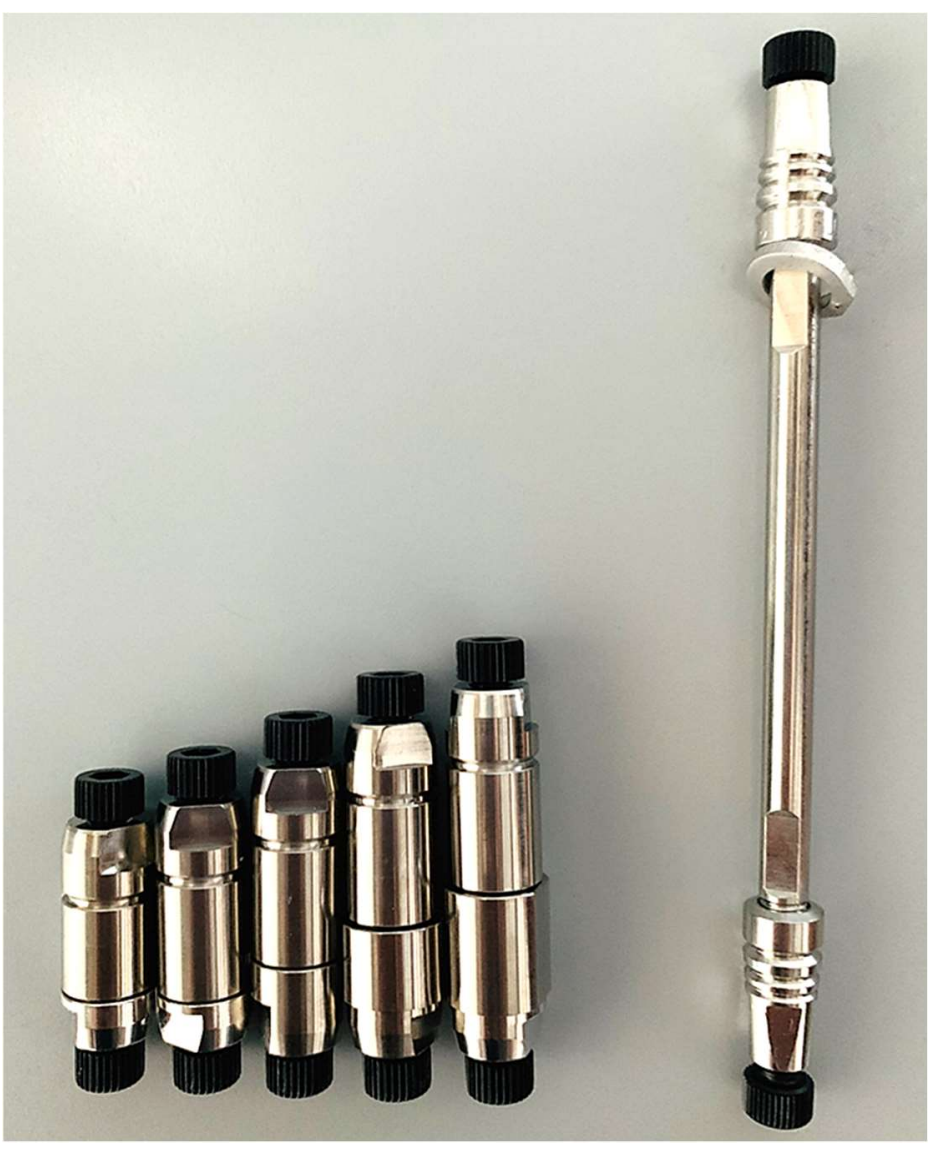

Supplementary Figure 1. CAD drawings of the new column hardware design $(A)$ and photo of the 2, 5, 10, 15 and $20 \mathrm{~mm}$ long prototype columns in comparison with a commercial $50 \times 2.1$ mm column (B). 
(A)

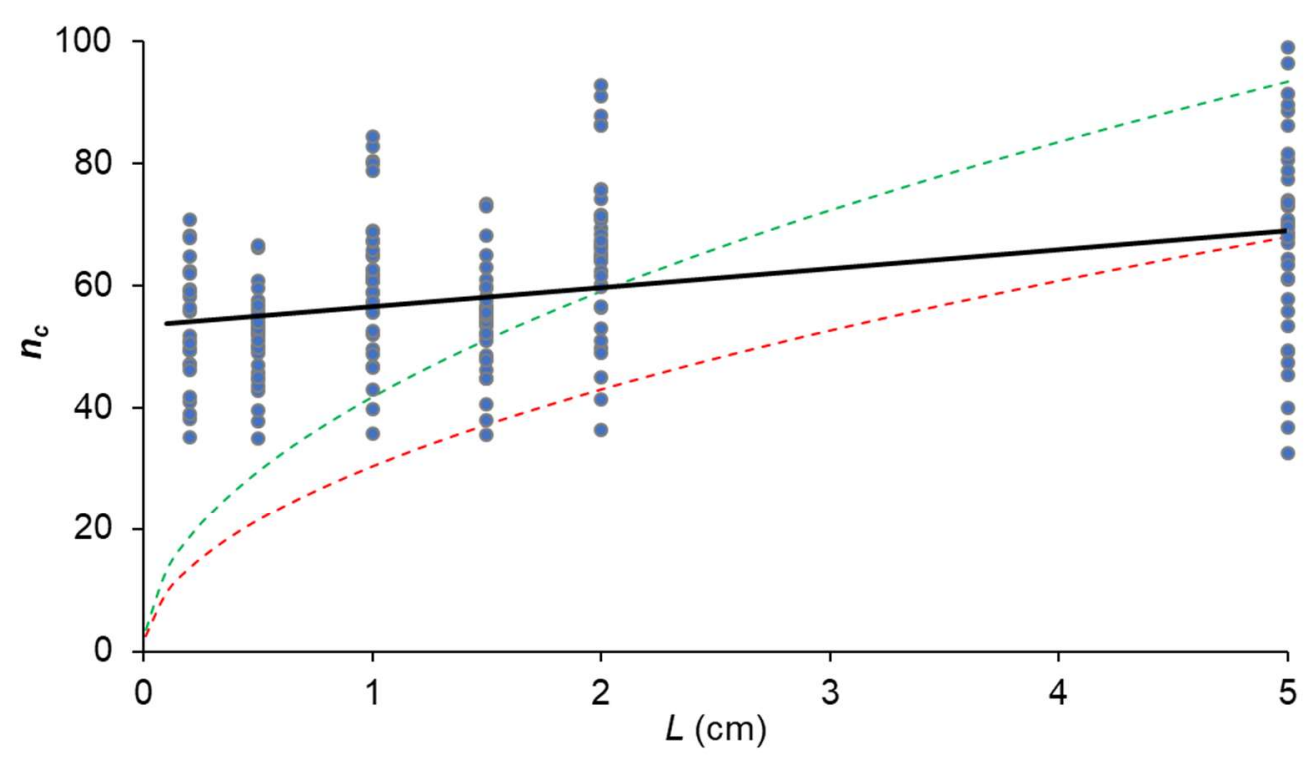

(B)

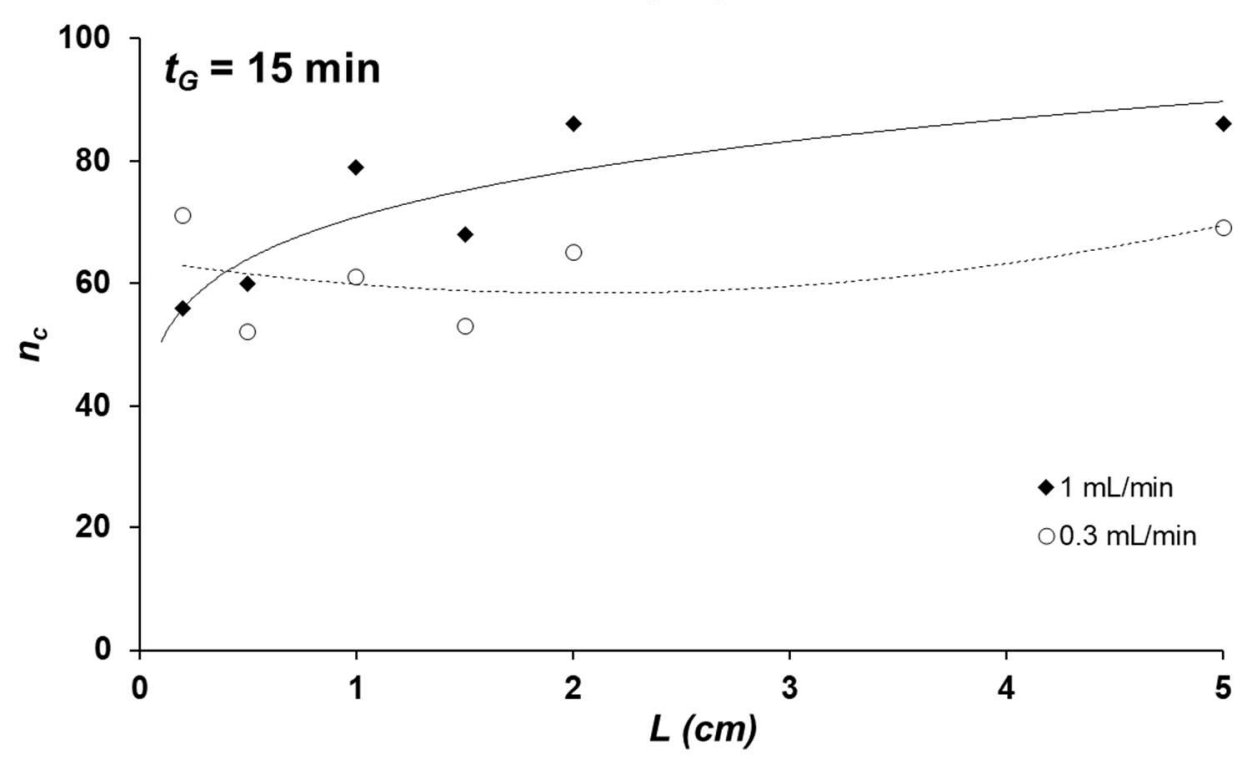

Supplementary Figure 2. Experimentally measured peak capacity values (peak capacity range obtainable in a practically interesting range of $t_{G}$ and $F$ ) as a function of column length taking into account all conditions (all $t_{G}$ and $F$ ) $-(A)$ and representative examples on the evolution of peak capacity vs. column length at $t_{G}=15 \mathrm{~min}$ and at $F_{1}=1 \mathrm{~mL} / \mathrm{min}$ and $F_{2}=0.3$ $\mathrm{mL} / \mathrm{min}(\mathrm{B})$.

On panel (A), a regression line (black line) indicates the trend of the experimental data. The red and green dashed lines corresponds to the peak capacity of 5 and $2 \mathrm{~cm}$ long columns. These two curves were constructed based on experimental peak capacities obtained on 5 and $2 \mathrm{~cm}$ columns, respectively, and extrapolated down to a column length $0 \mathrm{~cm}$ by calculation of peak capacity values proportional to the square root of column length.

Based on linear regression (black line), the lower and upper confidence limits $(95 \%)$ of intercept were 50.9 and 56.0 , respectively (significantly different from $0, p=2.7 \times 10^{-99}$ ). The lower and upper confidence limits (95\%) of the slope were 2.0 and 4.2 (significantly different from $\left.0, p=3.7 \times 10^{-8}\right)$. 
(A)

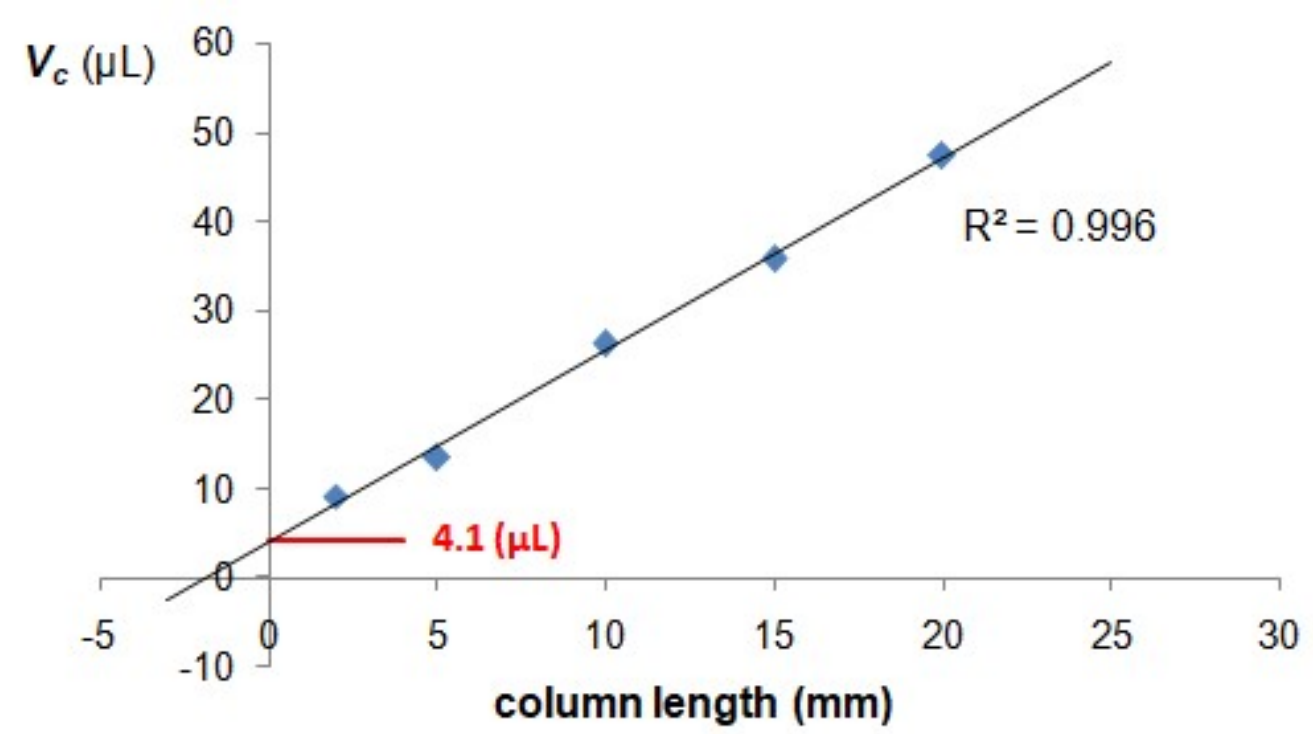

(B)

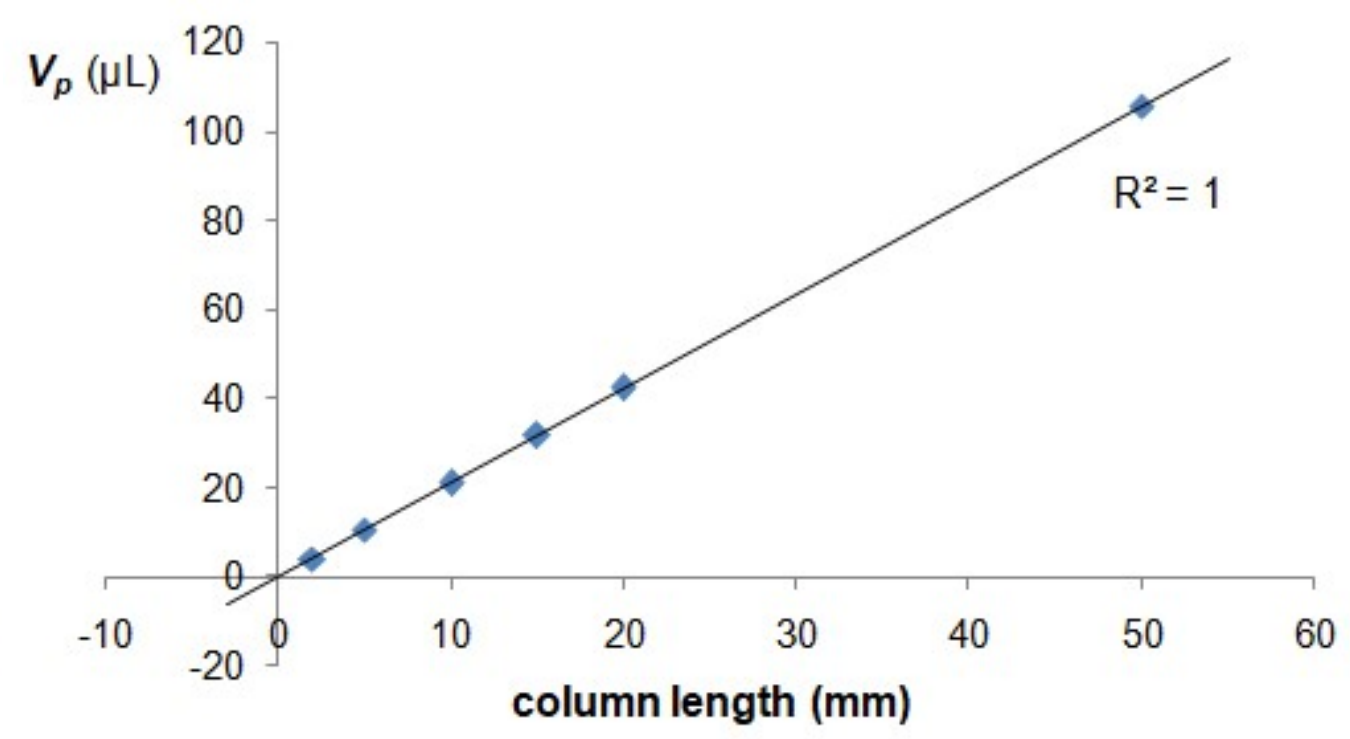

Supplementary Figure 3. Measured column volume $(A)$ and packed bed volume $(B)$ as a function of column length. 


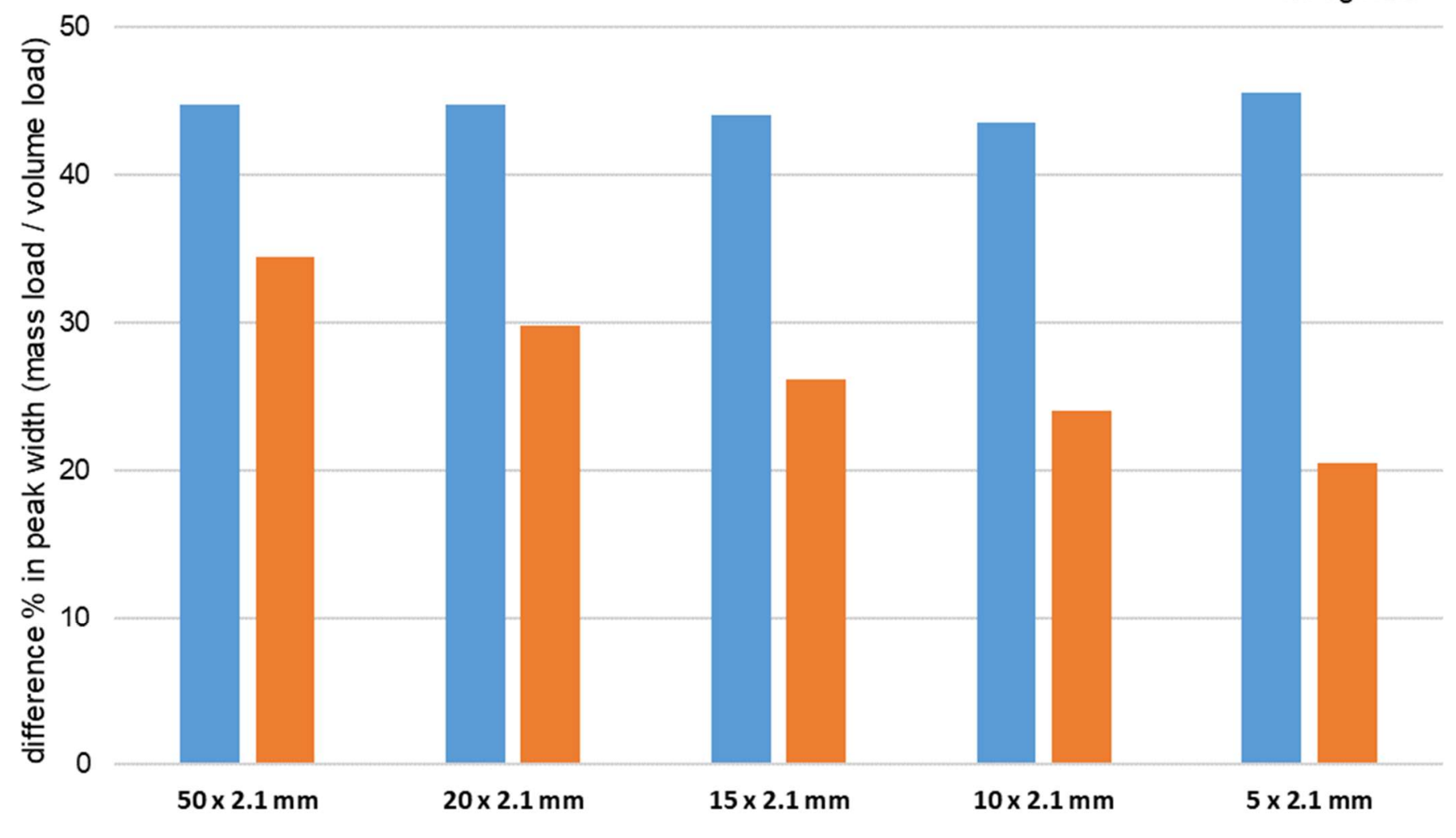

Supplementary Figure 4. Comparison of peak widths obtained by loading $\sim 1 \mu \mathrm{g}$ (blue bars) and $\sim 0.1 \mu \mathrm{g}$ (orange bars) samples. In case of $\sim 1 \mu \mathrm{g}$ load, 0.1 and $5 \mu \mathrm{L}$ injection volumes, while for the $\sim 0.1 \mu \mathrm{g}$ load, 0.1 and $1 \mu \mathrm{L}$ injection volumes are compared. The results are presented as \% peak width (low volume injection / high volume injection). Sample: light-chain fragment of pembrolizumab. 
Supplementary Table 1. retention time and peak width data of intact pembrolizumab

\begin{tabular}{|c|c|c|c|c|c|c|c|c|c|c|c|c|c|}
\hline \multirow[b]{3}{*}{$\begin{array}{l}\text { Flow rate } \\
(\mathrm{mL} / \mathrm{min})\end{array}$} & \multirow[b]{3}{*}{$\begin{array}{c}\text { Gradient } \\
\text { time }(\mathrm{min})\end{array}$} & \multicolumn{12}{|c|}{ Pembrolizumab } \\
\hline & & \multicolumn{2}{|c|}{$50 \times 2.1 \mathrm{~mm}$} & \multicolumn{2}{|c|}{$20 \times 2.1 \mathrm{~mm}$} & \multicolumn{2}{|c|}{$15 \times 2.1 \mathrm{~mm}$} & \multicolumn{2}{|c|}{$10 \times 2.1 \mathrm{~mm}$} & \multicolumn{2}{|c|}{$5 \times 2.1 \mathrm{~mm}$} & \multicolumn{2}{|c|}{$2 \times 2.1 \mathrm{~mm}$} \\
\hline & & Rt & $w 50 \%$ & Rt & w50\% & Rt & w50\% & Rt & w50\% & Rt & w50\% & Rt & w50\% \\
\hline \multirow{7}{*}{0.3} & 2 & 1.651 & 3.06E-02 & 1.4 & 3.06E-02 & 1.269 & $3.21 \mathrm{E}-02$ & 1.339 & $3.23 E-02$ & 1.183 & 3.38E-02 & 1.191 & 3.39E-02 \\
\hline & 3.5 & 2.303 & 4.16E-02 & 2.02 & 4.03E-02 & 1.826 & 4.55E-02 & 1.97 & 4.39E-02 & 1.736 & 4.86E-02 & 1.793 & $4.22 \mathrm{E}-02$ \\
\hline & 5 & 2.976 & 5.20E-02 & 2.655 & 5.15E-02 & 2.402 & 6.09E-02 & 2.622 & 5.67E-02 & 2.314 & 6.51E-02 & 2.383 & 5.34E-02 \\
\hline & 10 & 4.996 & 8.94E-02 & 4.623 & $9.18 \mathrm{E}-02$ & 4.138 & 1.15E-01 & 4.631 & $1.01 \mathrm{E}-01$ & 4.038 & $1.20 \mathrm{E}-01$ & 4.198 & 1.00E-01 \\
\hline & 15 & 7.018 & 1.27E-01 & 6.543 & 1.37E-01 & 5.901 & 1.67E-01 & 6.615 & 1.47E-01 & 5.824 & 1.73E-01 & 6.105 & 1.26E-01 \\
\hline & 20 & 8.963 & 1.66E-01 & 8.41 & $1.82 \mathrm{E}-01$ & 7.568 & 2.22E-01 & 8.547 & 1.94E-01 & 7.516 & 2.29E-01 & $N A$ & $N A$ \\
\hline & 25 & 10.91 & 2.11E-01 & 10.274 & $2.28 \mathrm{E}-01$ & 9.24 & 2.76E-01 & 10.475 & 2.45E-01 & 9.217 & 2.85E-01 & $N A$ & $N A$ \\
\hline \multirow{7}{*}{0.4} & 2 & 1.499 & 2.84E-02 & 1.282 & 2.73E-02 & 1.171 & 3.03E-02 & 1.235 & 2.94E-02 & 1.101 & 3.13E-02 & 1.096 & $3.13 \mathrm{E}-02$ \\
\hline & 3.5 & 2.189 & 3.93E-02 & 1.921 & 3.82E-02 & 1.755 & 4.45E-02 & 1.888 & 4.15E-02 & 1.68 & 4.68E-02 & 1.712 & 4.09E-02 \\
\hline & 5 & 2.851 & 4.89E-02 & 2.547 & 4.85E-02 & 2.33 & 5.78E-02 & 2.525 & 5.29E-02 & 2.252 & 6.07E-02 & 2.296 & $5.12 \mathrm{E}-02$ \\
\hline & 10 & 4.938 & 8.51E-02 & 4.523 & 8.85E-02 & 4.123 & 1.10E-01 & 4.548 & $9.60 \mathrm{E}-02$ & 4.039 & 1.14E-01 & 4.125 & $9.55 \mathrm{E}-02$ \\
\hline & 15 & 7.011 & 1.20E-01 & 6.476 & $1.28 \mathrm{E}-01$ & 5.902 & 1.59E-01 & 6.555 & 1.38E-01 & 5.826 & 1.66E-01 & 5.981 & $1.31 \mathrm{E}-01$ \\
\hline & 20 & 9.068 & 1.66E-01 & 8.395 & $1.74 \mathrm{E}-01$ & 7.638 & 2.11E-01 & 8.545 & 1.90E-01 & 7.573 & 2.18E-01 & $N A$ & $N A$ \\
\hline & 25 & 11.24 & $2.20 \mathrm{E}-01$ & 10.354 & 2.32E-01 & 9.37 & 2.72E-01 & 10.599 & 2.53E-01 & 9.319 & 2.83E-01 & $N A$ & $N A$ \\
\hline \multirow{7}{*}{0.5} & 2 & 1.383 & 2.68E-02 & 1.217 & 2.54E-02 & 1.126 & 2.86E-02 & 1.187 & 2.72E-02 & 1.072 & 3.00E-02 & 1.058 & 2.91E-02 \\
\hline & 3.5 & 2.124 & 3.52E-02 & 1.85 & 3.60E-02 & 1.71 & 4.22E-02 & 1.832 & 3.93E-02 & 1.653 & 4.44E-02 & 1.63 & 4.43E-02 \\
\hline & 5 & 2.799 & 4.51E-02 & 2.464 & 4.69E-02 & 2.281 & 5.67E-02 & 2.464 & 5.17E-02 & 2.222 & 5.96E-02 & 2.193 & 6.02E-02 \\
\hline & 10 & 5 & 7.53E-02 & 4.487 & 8.34E-02 & 4.151 & 1.05E-01 & 4.544 & 9.15E-02 & 4.101 & 1.10E-01 & 4.151 & 8.80E-02 \\
\hline & 15 & 7.156 & 1.19E-01 & 6.431 & $1.24 \mathrm{E}-01$ & 5.939 & $1.53 \mathrm{E}-01$ & 6.552 & 1.36E-01 & 5.892 & $1.60 \mathrm{E}-01$ & 5.897 & 1.45E-01 \\
\hline & 20 & 9.386 & 1.60E-01 & 8.439 & $1.79 \mathrm{E}-01$ & 7.728 & 2.16E-01 & 8.656 & 1.96E-01 & 7.69 & 2.26E-01 & $N A$ & $N A$ \\
\hline & 25 & 11.351 & 1.83E-01 & 10.391 & $2.08 \mathrm{E}-01$ & 9.529 & 2.71E-01 & 10.644 & 2.22E-01 & 9.494 & 2.83E-01 & $N A$ & $N A$ \\
\hline \multirow{7}{*}{0.7} & 2 & 1.324 & 2.33E-02 & 1.133 & 2.36E-02 & 1.054 & 2.60E-02 & 1.108 & $2.52 E-02$ & 1.015 & $2.72 \mathrm{E}-02$ & 0.986 & 2.85E-02 \\
\hline & 3.5 & 2.033 & 3.27E-02 & 1.769 & 3.42E-02 & 1.645 & 3.95E-02 & 1.754 & 3.72E-02 & 1.609 & 4.19E-02 & 1.563 & 4.45E-02 \\
\hline & 5 & 2.726 & 4.15E-02 & 2.391 & 4.46E-02 & 2.226 & 5.29E-02 & 2.388 & 4.88E-02 & 2.188 & 5.62E-02 & 2.129 & 6.04E-02 \\
\hline & 10 & 4.981 & 7.18E-02 & 4.416 & 7.80E-02 & 4.105 & 9.74E-02 & 4.457 & 8.62E-02 & 4.092 & 1.04E-01 & 4.06 & $9.20 \mathrm{E}-02$ \\
\hline & 15 & 7.267 & 9.97E-02 & 6.457 & $1.20 \mathrm{E}-01$ & 5.962 & $1.55 \mathrm{E}-01$ & 6.564 & 1.33E-01 & 5.952 & 1.66E-01 & 5.847 & $1.52 \mathrm{E}-01$ \\
\hline & 20 & 9.387 & 1.19E-01 & 8.477 & 1.37E-01 & 7.891 & 2.00E-01 & 8.615 & 1.48E-01 & 7.87 & 2.17E-01 & $N A$ & $N A$ \\
\hline & 25 & 11.438 & 1.53E-01 & 10.319 & 1.69E-01 & 9.641 & 2.29E-01 & 10.508 & 1.86E-01 & 9.598 & $2.46 \mathrm{E}-01$ & $N A$ & $N A$ \\
\hline \multirow{7}{*}{1} & 2 & 1.233 & 2.28E-02 & 1.062 & $2.28 \mathrm{E}-02$ & 0.995 & 2.46E-02 & 1.035 & 2.41E-02 & 0.942 & 2.64E-02 & 0.917 & 3.07E-02 \\
\hline & 3.5 & 1.942 & 3.31E-02 & 1.695 & 3.35E-02 & 1.593 & 3.72E-02 & 1.677 & 3.61E-02 & 1.524 & 4.09E-02 & 1.497 & 4.54E-02 \\
\hline & 5 & 2.633 & 4.29E-02 & 2.314 & 4.39E-02 & 2.176 & 4.97E-02 & 2.297 & 4.75E-02 & 2.093 & 5.52E-02 & 2.079 & 5.78E-02 \\
\hline & 10 & 4.91 & 7.47E-02 & 4.35 & 7.85E-02 & 4.073 & 9.45E-02 & 4.36 & 8.66E-02 & 3.94 & 1.05E-01 & 3.884 & 1.19E-01 \\
\hline & 15 & 7.09 & 1.03E-01 & 6.332 & $1.03 \mathrm{E}-01$ & 5.948 & 1.31E-01 & 6.366 & 1.13E-01 & 5.785 & $1.50 \mathrm{E}-01$ & 5.762 & $1.59 \mathrm{E}-01$ \\
\hline & 20 & 9.253 & 1.32E-01 & 8.264 & 1.30E-01 & 7.772 & 1.62E-01 & 8.333 & 1.44E-01 & 7.604 & $1.81 \mathrm{E}-01$ & $N A$ & $N A$ \\
\hline & 25 & 11.431 & $1.62 E-01$ & 10.171 & $1.60 \mathrm{E}-01$ & 9.55 & 2.04E-01 & 10.279 & 1.76E-01 & 9.366 & $2.24 \mathrm{E}-01$ & $N A$ & $N A$ \\
\hline
\end{tabular}


Supplementary Table 2. retention time and peak width data of reduced pembrolizumab (LC: light chain peak, $\mathrm{HC}$ : heavy chain peak)

\begin{tabular}{|c|c|c|c|c|c|c|c|c|c|c|c|c|c|c|c|c|c|c|c|c|c|c|c|c|c|}
\hline \multirow{4}{*}{$\begin{array}{l}\text { Flow rate } \\
(\mathrm{m} \sqcup \mathrm{min})\end{array}$} & \multirow[b]{4}{*}{$\begin{array}{l}\text { Gradient } \\
\text { time (min) }\end{array}$} & \\
\hline & & \multirow{2}{*}{\multicolumn{2}{|c|}{$\begin{array}{c}\text { LC } \\
50 \times 2.1 \mathrm{~mm}\end{array}$}} & \multirow{2}{*}{\multicolumn{2}{|c|}{$\begin{array}{c}\mathrm{HC} \\
50 \times 2.1 \mathrm{~mm}\end{array}$}} & \multirow{2}{*}{\multicolumn{2}{|c|}{$\begin{array}{c}\text { LC } \\
20 \times 2.1 \mathrm{~mm}\end{array}$}} & \multirow{2}{*}{\multicolumn{2}{|c|}{$\begin{array}{c}\mathrm{HC} \\
20 \times 2.1 \mathrm{~mm}\end{array}$}} & \multirow{2}{*}{\multicolumn{2}{|c|}{$\begin{array}{c}\text { LC } \\
15 \times 2.1 \mathrm{~mm}\end{array}$}} & \multirow{2}{*}{\multicolumn{2}{|c|}{$\begin{array}{c}\mathrm{HC} \\
15 \times 2.1 \mathrm{~mm}\end{array}$}} & \multirow{2}{*}{\multicolumn{2}{|c|}{$\begin{array}{c}L C \\
10 \times 2.1 \mathrm{~mm}\end{array}$}} & \multirow{2}{*}{\multicolumn{2}{|c|}{$\begin{array}{c}\mathrm{HC} \\
10 \times 2.1 \mathrm{~mm}\end{array}$}} & \multirow{2}{*}{\multicolumn{2}{|c|}{$\begin{array}{c}\text { LC } \\
5 \times 2.1 \mathrm{~mm}\end{array}$}} & \multirow{2}{*}{\multicolumn{2}{|c|}{$\begin{array}{c}\mathrm{HC} \\
5 \times 2.1 \mathrm{~mm}\end{array}$}} & \multirow{2}{*}{\multicolumn{2}{|c|}{$\begin{array}{c}\mathrm{LC} \\
2 \times 2.1 \mathrm{~mm}\end{array}$}} & \multirow{2}{*}{\multicolumn{2}{|c|}{$\begin{array}{c}\mathrm{HC} \\
2 \times 2.1 \mathrm{~mm}\end{array}$}} \\
\hline & & & & & & & & & & & & & & & & & & & & & & & & & \\
\hline & & $\mathrm{Rt}$ & w50\% & Rt & w50\% & Rt & w50\% & Rt & w50\% & Rt & w50\% & Rt & w50\% & Rt & w50\% & Rt & w50\% & Rt & w50\% & Rt & w50\% & $\mathrm{Rt}$ & w50\% & Rt & w50\% \\
\hline \multirow{7}{*}{0.3} & 2 & 1.52 & $\mathrm{NA}$ & 1.572 & $3.26 \mathrm{E}-02$ & 1.206 & $2.70 \mathrm{E}-02$ & 1.289 & $2.71 \mathrm{E}-02$ & 1.027 & $2.85 \mathrm{E}-02$ & 1.145 & $2.93 \mathrm{E}-02$ & 1.078 & $3.13 \mathrm{E}-02$ & 1.188 & $3.11 \mathrm{E}-02$ & 0.85 & 3.72E-02 & 1.009 & $3.88 \mathrm{E}-02$ & 0.871 & $3.56 \mathrm{E}-02$ & 1.039 & $3.29 \mathrm{E}-02$ \\
\hline & 3.5 & 1.996 & $3.79 \mathrm{E}-02$ & 2.145 & 4.12E-02 & 1.607 & $3.71 \mathrm{E}-02$ & 1.801 & $3.73 \mathrm{E}-02$ & 1.328 & 4.13E-02 & 1.583 & $4.23 \mathrm{E}-02$ & 1.449 & 4.41E-02 & 1.685 & 4.37E-02 & 1.075 & $5.50 \mathrm{E}-02$ & 1.402 & $5.69 \mathrm{E}-02$ & 1.188 & $4.92 \mathrm{E}-02$ & 1.519 & $4.40 \mathrm{E}-02$ \\
\hline & 5 & 2.465 & 4.74E-02 & 2.73 & $5.08 \mathrm{E}-02$ & 2.009 & $4.84 \mathrm{E}-02$ & 2.333 & $4.92 \mathrm{E}-02$ & 1.621 & $5.48 \mathrm{E}-02$ & 2.03 & $5.68 \mathrm{E}-02$ & 1.817 & $5.75 E-02$ & 2.196 & $5.72 \mathrm{E}-02$ & 1.298 & $7.30 \mathrm{E}-02$ & 1.806 & 7.61E-02 & 1.481 & $6.36 \mathrm{E}-02$ & 1.984 & $5.54 \mathrm{E}-02$ \\
\hline & 10 & 3.746 & $7.76 \mathrm{E}-02$ & 4.433 & $8.41 \mathrm{E}-02$ & 3.119 & $8.37 \mathrm{E}-02$ & 3.889 & $8.80 \mathrm{E}-02$ & 2.421 & $9.58 \mathrm{E}-02$ & 3.338 & $1.04 \mathrm{E}-01$ & 2.867 & $9.79 \mathrm{E}-02$ & 3.724 & 1.01E-01 & 1.924 & $1.22 \mathrm{E}-01$ & 3.003 & 1.34E-01 & 2.265 & $1.05 \mathrm{E}-01$ & 3.333 & 9.67E-02 \\
\hline & 15 & 4.997 & 1.07E-01 & 6.135 & $1.18 \mathrm{E}-01$ & 4.168 & 1.19E-01 & 5.419 & $1.28 \mathrm{E}-01$ & 3.14 & 1.37E-01 & 4.612 & 1.49E-01 & 3.812 & $1.38 \mathrm{E}-01$ & 5.196 & $1.45 \mathrm{E}-01$ & 2.418 & 1.82E-01 & 4.141 & 1.94E-01 & 2.966 & 1.45E-01 & 4.684 & 1.29E-01 \\
\hline & 20 & 6.0 & $1.40 \mathrm{E}-$ & 7.7 & 52E-C & 5. & $60 \mathrm{E}-$ & & $69 \mathrm{E}$ & 3.662 & 1.80 & 5.7 & 1.97 & 4.6 & & 6.5 & & & & & & & & & \\
\hline & 25 & 7. & & 9.2 & & 50 & 1 & & & 4.2 & & & & & & 7.9 & & & & & & & & & \\
\hline \multirow{7}{*}{0.4} & 2 & 1.337 & $2.75 \mathrm{E}-02$ & 1.402 & 2.82E-02 & 1.067 & $2.64 \mathrm{E}-02$ & 1.159 & $2.58 \mathrm{E}-02$ & 0.907 & $2.85 \mathrm{E}-02$ & 1.034 & 2.86E-02 & 0.956 & $.03 \mathrm{E}-02$ & 1.075 & 2.94E-02 & 0.745 & 3.55E-02 & 0.915 & 3.64E-02 & 0.773 & 3.36E-02 & 0.95 & 3.19E-02 \\
\hline & 3.5 & 1.828 & $3.61 \mathrm{E}-02$ & 1.998 & $3.67 \mathrm{E}-02$ & 1.483 & $3.75 \mathrm{E}-02$ & 1.693 & $3.66 \mathrm{E}-02$ & 1.22 & 4.19E-02 & 1.49 & $4.19 \mathrm{E}-02$ & 1.334 & $4.41 \mathrm{E}-02$ & 1.585 & $4.24 \mathrm{E}-02$ & 0.982 & $5.39 \mathrm{E}-02$ & 1.324 & $5.48 \mathrm{E}-02$ & 1.086 & $5.22 \mathrm{E}-02$ & 1.431 & 4.47E-02 \\
\hline & 5 & 2.29 & $4.48 \mathrm{E}-02$ & 2.572 & 4.51E-02 & 1.885 & $4.82 \mathrm{E}-02$ & 2.216 & 4.71E-02 & 1.52 & $5.52 \mathrm{E}-02$ & 1.939 & $5.45 \mathrm{E}-02$ & 1.7 & $5.69 \mathrm{E}-02$ & 2.087 & $5.44 \mathrm{E}-02$ & 1.212 & $7.22 \mathrm{E}-02$ & 1.732 & $7.24 \mathrm{E}-02$ & 1.365 & & 1.868 & $5.52 \mathrm{E}-02$ \\
\hline & 10 & 3.591 & 7.43E-02 & 4.315 & 7.47E-02 & 2.97 & $8.53 \mathrm{E}-02$ & 3.774 & $8.59 \mathrm{E}-02$ & 2.294 & $9.86 \mathrm{E}-02$ & 3.257 & $1.01 \mathrm{E}-01$ & 2.701 & $9.92 \mathrm{E}-02$ & 3.6 & $9.70 \mathrm{E}-02$ & 1.783 & $1.26 \mathrm{E}-01$ & 2.919 & 1.33E-01 & 2.102 & 1.14E-01 & 3.218 & $9.83 \mathrm{E}-02$ \\
\hline & 15 & 4.805 & $1.03 \mathrm{E}-01$ & 6.007 & $1.04 \mathrm{E}-01$ & 3.993 & $1.21 \mathrm{E}-01$ & 5.298 & $1.23 \mathrm{E}-01$ & 3.013 & $1.38 \mathrm{E}-01$ & 4.542 & $1.45 \mathrm{E}-01$ & 3.636 & $1.40 \mathrm{E}-01$ & 5.076 & $1.39 \mathrm{E}-01$ & 2.317 & $1.76 \mathrm{E}-01$ & 4.079 & 1.91 & 2.787 & $1.56 \mathrm{E}-01$ & 4.545 & $1.35 \mathrm{E}-01$ \\
\hline & 20 & 5013 & D & 7.6 & & 4.913 & 575 & 6.7 & $1.61 \mathrm{E}-01$ & 3.60 & 1.8 & & & & & 6.482 & & & & & & & $N A$ & A & $N A$ \\
\hline & 25 & & & & & & & & & & & & & & & & & & & & & & & & \\
\hline & 2 & 1.244 & & 1.3 & & & & & & & & & & & & 1.0 & & & & & & & & 302 & $=-02$ \\
\hline & 3.5 & 1.727 & 2 & 1.908 & 2 & 1.401 & $3.70 \mathrm{E}$ & 1.6 & 55 & 1.164 & 4.11 & 1.437 & & 1.263 & & 1.52 & & 0.943 & & 1.286 & & 1.014 & $5.42 \mathrm{E}-02$ & 1.358 & 4.51E-02 \\
\hline & 5 & 2. & & 2.4 & 4.3 & 1.7 & & & 4. & 1.438 & 5.55 & 1.868 & & & & & & 1.149 & & 1.674 & & 2.037 & 1.20E-01 & 3.171 & $1.00 \mathrm{E}-01$ \\
\hline 0.5 & 10 & 3.531 & 7.4 & 4.285 & 7.2 & 2.9 & 2 & & 8. & 2.27 & 9.95 & 3.2 & & 2.631 & & 3.551 & & 1.783 & & 2.932 & & 1.265 & & 1.776 & $6.08 \mathrm{E}-02$ \\
\hline & 15 & 4.728 & $1.02 \mathrm{E}$ & 5.974 & $9.98 \mathrm{E}$ & 3.8 & 1.24 & 5.228 & 1. & 2.9 & 1.43 & 4.5 & & 3.529 & & & & & & 4.088 & & 2.676 & $1.73 \mathrm{E}-01$ & 4.454 & $1.48 \mathrm{E}-01$ \\
\hline & 20 & 5.85 & & 7.62 & & & & 6.7 & & 3.5 & & & & & & & & & & & & $N$ & $N A$ & $N A$ & $N A$ \\
\hline & 25 & & & 9.2 & & & & 8.147 & & & & & & & & & & & & & & & $N A$ & $N A$ & $N A$ \\
\hline & -1 & & & & & & & & & & & & & & & & & & & & & & & 36 & \\
\hline & 3.5 & 1.585 & & 1. & & & & & & & & & & & & & & & & & & & & & $=-02$ \\
\hline & 5 & 2. & 4. & 2.3 & & 1.6 & & 2. & & & & & & & & & & & & & & & & & 6.3 \\
\hline 0.7 & 10 & 3.5 & & 4.2 & & & & & & & & & & & & & & & & & & & & 4 & $1.08 \mathrm{E}-01$ \\
\hline & 15 & & & & & & & & & & & & & & & & & & & & & 2.535 & 1.90E-01 & 4.347 & $1.58 \mathrm{E}-01$ \\
\hline & 20 & 5.711 & 1.3 & 7.6 & $1.33 \mathrm{E}$ & 4.6 & 1. & 6.6 & 1.56 & 3.5 & 1.8 & 5.7 & & 4. & 1.93 & 6.2 & & 2.631 & 2.30 & 5.176 & & NA & $N A$ & $N A$ & $N A$ \\
\hline & 25 & 6.769 & 1.5 & 9.2 & 1.49E-01 & 5.503 & $1.96 \mathrm{E}-01$ & 8.063 & 1.92E-01 & 4.076 & 2.32 & 6.957 & & 4.931 & & 7.679 & & 3.017 & & & & $N_{t}$ & $N A$ & $N A$ & $N A$ \\
\hline & - & 0.988 & 2.22E-02 & 1.095 & $2.24 \mathrm{E}-02$ & 0.797 & 2.36E-02 & 0.921 & $2.30 \mathrm{E}-02$ & 0.674 & $2.62 \mathrm{E}-02$ & 0.832 & 22 & 0.709 & 2.71 & 0.857 & & 0.541 & 3.27 & 0.739 & 3.26E-02 & 0.566 & $85 \mathrm{E}-02$ & 0.762 & $34 \mathrm{E}-02$ \\
\hline & 3.5 & & & & & & & & & & & & & & & & & & & & & & & & $.10 \mathrm{E}-02$ \\
\hline & 5 & 1.8 & & & & & & & & & & & & & & & & & & & & & & & \\
\hline 1 & 10 & & & 4.0 & & & & & & & & & & & & & & & & & & & & & \\
\hline & 15 & & & & & & & & & & & & & & & & & & & & & 2.328 & 2.10E-01 & 4.196 & 1.73E-01 \\
\hline & 20 & 5.493 & $1.38 \mathrm{E}-01$ & 7.528 & UE- & 4.43 & $1.65 \mathrm{E}-01$ & 6.526 & $1.48 \mathrm{E}-01$ & 3.334 & $1.88 \mathrm{E}-01$ & 5.661 & & 3.901 & $44-01$ & 6.132 & & 2.4 & $2.42 \mathrm{E}-01$ & 5. & 2.4 & $N A$ & $N A$ & $N A$ & $N A$ \\
\hline & 25 & 6.58 & 1.73E-01 & 9.165 & 1.43E-01 & 5.274 & $2.00 \mathrm{E}-01$ & 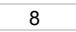 & 1.66E-01 & 3.907 & $2.29 \mathrm{E}-01$ & 6.929 & $2.30 \mathrm{E}-01$ & 4.63 & $2.38 \mathrm{E}-01$ & 7.55 & 2.12E-01 & 2.829 & $2.95 \mathrm{E}-01$ & 6.149 & 3.10E-01 & $N A$ & $N A$ & $N A$ & $N A$ \\
\hline
\end{tabular}


Supplementary Table 3. Data used to construct Figure 1: peak width $\left(w_{1 / 2}\right)$, gradient steepness $(b)$, relative peak width, peak capacity $\left(n_{c}\right)$ and peak capacity per pressure and time unit $\left(n_{c} / P^{*} t\right)$. All data were obtained by injecting intact pembrolizumab.

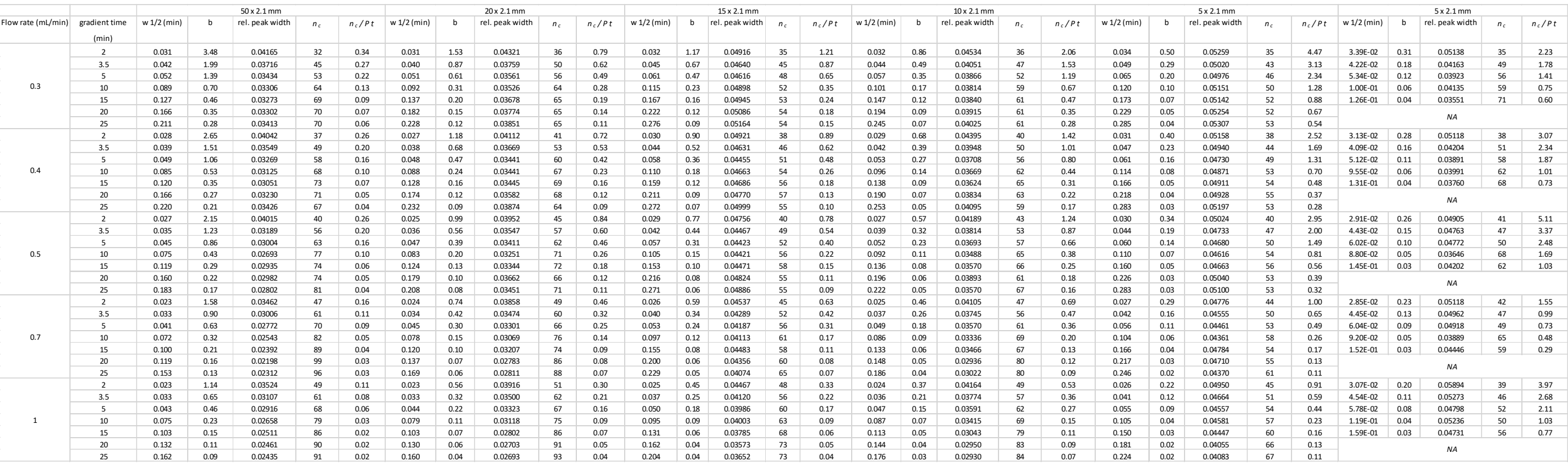


Supplementary Table 4. Data obtained during the loadability study. Sample: light-chain fragment of pembrolizumab.

\begin{tabular}{|c|c|c|c|c|c|c|c|c|}
\hline \multirow[b]{2}{*}{ Column } & \multirow[b]{2}{*}{ column volume (uL) } & mass load & volumetric load & mass load & volumetric load & mass load & volumetric load & \multirow{2}{*}{$\begin{array}{l}\text { volumetric load } \\
\text { inj. / column volume }\end{array}$} \\
\hline & & \multicolumn{2}{|c|}{ injected mass ( $\mu \mathrm{g})$} & \multicolumn{2}{|c|}{ injected mass / column volume (ug/uL) } & \multicolumn{2}{|c|}{ w50\% } & \\
\hline \multirow{6}{*}{$50 \times 2.1 \mathrm{~mm}$} & \multirow{6}{*}{105.7} & $1.000 \mathrm{E}+00$ & $5.000 \mathrm{E}+00$ & 9.461E-03 & 4.730E-02 & $1.870 \mathrm{E}-02$ & 4.193E-02 & $9.461 \mathrm{E}-04$ \\
\hline & & 1.000E-01 & $2.000 \mathrm{E}+00$ & 9.461E-04 & 1.892E-02 & $1.850 \mathrm{E}-02$ & 4.133E-02 & 1.892E-03 \\
\hline & & 5.000E-02 & $1.000 \mathrm{E}+00$ & 4.730E-04 & $9.461 \mathrm{E}-03$ & 1.844E-02 & 4.173E-02 & 4.730E-03 \\
\hline & & 2.500E-02 & $5.000 \mathrm{E}-01$ & 2.365E-04 & 4.730E-03 & 1.855E-02 & 4.279E-02 & $9.461 \mathrm{E}-03$ \\
\hline & & 1.250E-02 & 2.000E-01 & 1.183E-04 & 1.892E-03 & 1.851E-02 & 4.531E-02 & 1.892E-02 \\
\hline & & 6.250E-03 & 1.000E-01 & 5.913E-05 & $9.461 \mathrm{E}-04$ & 1.826E-02 & 5.367E-02 & 4.730E-02 \\
\hline \multirow{6}{*}{$20 \times 2.1 \mathrm{~mm}$} & \multirow{6}{*}{42.3} & $1.000 \mathrm{E}+00$ & $5.000 \mathrm{E}+00$ & 2.364E-02 & 1.182E-01 & 1.993E-02 & 4.656E-02 & 2.364E-03 \\
\hline & & 1.000E-01 & $2.000 \mathrm{E}+00$ & 2.364E-03 & 4.728E-02 & 1.950E-02 & 4.391E-02 & 4.728E-03 \\
\hline & & 5.000E-02 & $1.000 \mathrm{E}+00$ & 1.182E-03 & 2.364E-02 & 1.948E-02 & 4.451E-02 & 1.182E-02 \\
\hline & & 2.500E-02 & $5.000 \mathrm{E}-01$ & 5.910E-04 & 1.182E-02 & 1.936E-02 & 4.650E-02 & 2.364E-02 \\
\hline & & 1.250E-02 & $2.000 \mathrm{E}-01$ & 2.955E-04 & 4.728E-03 & 1.932E-02 & 5.117E-02 & 4.728E-02 \\
\hline & & 6.250E-03 & 1.000E-01 & 1.478E-04 & 2.364E-03 & 1.993E-02 & 6.491E-02 & 1.182E-01 \\
\hline \multirow{6}{*}{$15 \times 2.1 \mathrm{~mm}$} & \multirow{6}{*}{31.7} & $1.000 E+00$ & $5.000 \mathrm{E}+00$ & 3.155E-02 & 1.577E-01 & 2.140E-02 & 4.771E-02 & 3.155E-03 \\
\hline & & 1.000E-01 & $2.000 \mathrm{E}+00$ & $3.155 \mathrm{E}-03$ & 6.309E-02 & 2.050E-02 & 4.711E-02 & $6.309 \mathrm{E}-03$ \\
\hline & & 5.000E-02 & $1.000 \mathrm{E}+00$ & 1.577E-03 & 3.155E-02 & 2.058E-02 & 4.860E-02 & 1.577E-02 \\
\hline & & 2.500E-02 & $5.000 \mathrm{E}-01$ & 7.886E-04 & 1.577E-02 & 2.031E-02 & $5.181 \mathrm{E}-02$ & 3.155E-02 \\
\hline & & 1.250E-02 & $2.000 \mathrm{E}-01$ & 3.943E-04 & 6.309E-03 & 1.996E-02 & $5.874 \mathrm{E}-02$ & 6.309E-02 \\
\hline & & 6.250E-03 & $1.000 \mathrm{E}-01$ & 1.972E-04 & $3.155 \mathrm{E}-03$ & 1.982E-02 & 7.637E-02 & 1.577E-01 \\
\hline \multirow{6}{*}{$10 \times 2.1 \mathrm{~mm}$} & \multirow{6}{*}{21.1} & $1.000 \mathrm{E}+00$ & $5.000 \mathrm{E}+00$ & 4.739E-02 & 2.370E-01 & 2.196E-02 & 4.904E-02 & 4.739E-03 \\
\hline & & 1.000E-01 & $2.000 \mathrm{E}+00$ & 4.739E-03 & 9.479E-02 & 2.131E-02 & 4.880E-02 & 9.479E-03 \\
\hline & & 5.000E-02 & $1.000 \mathrm{E}+00$ & 2.370E-03 & 4.739E-02 & 2.109E-02 & 5.041E-02 & 2.370E-02 \\
\hline & & 2.500E-02 & $5.000 \mathrm{E}-01$ & 1.185E-03 & $2.370 \mathrm{E}-02$ & 2.076E-02 & $5.420 \mathrm{E}-02$ & 4.739E-02 \\
\hline & & 1.250E-02 & $2.000 \mathrm{E}-01$ & 5.924E-04 & 9.479E-03 & 2.032E-02 & $6.242 \mathrm{E}-02$ & 9.479E-02 \\
\hline & & 6.250E-03 & 1.000E-01 & 2.962E-04 & 4.739E-03 & 2.082E-02 & 8.447E-02 & $2.370 \mathrm{E}-01$ \\
\hline \multirow{6}{*}{$5 \times 2.1 \mathrm{~mm}$} & \multirow{6}{*}{10.6} & $1.000 E+00$ & $5.000 \mathrm{E}+00$ & 9.434E-02 & 4.717E-01 & $2.555 \mathrm{E}-02$ & $5.235 \mathrm{E}-02$ & $9.434 \mathrm{E}-03$ \\
\hline & & 1.000E-01 & $2.000 \mathrm{E}+00$ & 9.434E-03 & 1.887E-01 & 2.378E-02 & $5.230 \mathrm{E}-02$ & 1.887E-02 \\
\hline & & 5.000E-02 & $1.000 \mathrm{E}+00$ & 4.717E-03 & 9.434E-02 & 2.365E-02 & 5.610E-02 & 4.717E-02 \\
\hline & & 2.500E-02 & $5.000 \mathrm{E}-01$ & 2.358E-03 & 4.717E-02 & 2.332E-02 & 6.380E-02 & $9.434 \mathrm{E}-02$ \\
\hline & & 1.250E-02 & 2.000E-01 & 1.179E-03 & 1.887E-02 & 2.335E-02 & 7.849E-02 & 1.887E-01 \\
\hline & & 6.250E-03 & $1.000 \mathrm{E}-01$ & 5.896E-04 & 9.434E-03 & 2.271E-02 & 1.142E-01 & 4.717E-01 \\
\hline
\end{tabular}

